\title{
Erratum to: On the Geometry of Cyclic Lattices
}

\author{
Lenny Fukshansky ${ }^{1}$. Xun Sun ${ }^{2}$
}

Published online: 22 April 2015

(C) Springer Science+Business Media New York 2015

\section{Erratum to: Discrete Comput Geom (2014) 52:240-259 DOI 10.1007/s00454-014-9608-3}

A mistake in the results of our previous paper is corrected.

Theorems 1.1 and 1.2 of our paper [1] are incorrect as stated. Specifically, these theorems assert that the well-rounded (WR) lattices comprise a positive proportion of all cyclic lattices in every dimension. This assertion is based on comparative asymptotic counting estimates for WR cyclic lattices versus all cyclic lattices with successive minima bounded by $R$ as $R \rightarrow \infty$. While the lower bound on the number of WR cyclic lattices produced in Sect. 4 is correct, the upper bound on the number of all cyclic lattices produced in Sect. 3 (specifically, Lemmas 3.2 and 3.3) is not accurate. Similarly, in the case $N=2$, there is a mistake in the estimate on the function $h_{2}(R)$ in the proof of Lemma 5.2, while the rest of Lemma 5.2 is correct. For the purposes of our current results, this means that WR cyclic lattices do not comprise a positive proportion of all cyclic lattices in a given dimension, and hence our results should instead be stated as counting estimates on the number of WR cyclic lattices. This

Editor in charge: Günter M. Ziegler

The online version of the original article can be found under doi:10.1007/s00454-014-9608-3.

Lenny Fukshansky

lenny@cmc.edu

Xun Sun

foxfur_32@hotmail.com

1 Department of Mathematics, Claremont McKenna College, 850 Columbia Avenue, Claremont, CA 91711, USA

2 School of Mathematical Sciences, Claremont Graduate University, Claremont, CA 91711, USA 
implies the following corrected statements of our theorems, which follow directly from the results of Sects. 4 and 5 of [1]. We write $\mathrm{WR}^{\prime}$ for lattices which are generated by their minimal vectors, a strictly stronger condition than WR in general.

Theorem 1.1, corrected. Let $R \in \mathbb{R}_{>0}$, then there exists a constant $\alpha_{N}>0$ depending only on dimension $N$ such that

$$
\#\left\{\Gamma \in \mathcal{C}_{N}: \lambda_{N}(\Gamma) \leq R, \Gamma \text { is } \mathrm{WR}^{\prime}\right\} \geq \alpha_{N} R^{N}
$$

as $R \rightarrow \infty$.

Proof This follows immediately from Lemma 4.3 of [1].

Theorem 1.2, corrected. Let $R \in \mathbb{R}_{>0}$, then

$$
\begin{aligned}
0.200650 \ldots \times R^{2}-3.742382 \ldots \times R & \leq \#\left\{\Gamma \in \mathcal{C}_{2}: \lambda_{2}(\Gamma) \leq R, \Gamma \text { is } \mathrm{WR}^{\prime}\right\} \\
& \leq 0.267638 \ldots \times R^{2}+1.673031 \ldots \times R
\end{aligned}
$$

Proof This follows immediately from Lemma 5.2 of [1].

Corollary 1.3, corrected. Let $N \geq 2$, let $\tau \in S_{N}$ be an $N$-cycle, and let $\mathcal{C}_{N}(\tau)$ be the set of all $\tau$-invariant full-rank sublattices of $\mathbb{Z}^{N}$. Then

$$
\#\left\{\Gamma \in \mathcal{C}_{N}(\tau): \lambda_{N}(\Gamma) \leq R, \Gamma \text { is } \mathrm{WR}^{\prime}\right\} \geq \alpha_{N} R^{N},
$$

as $R \rightarrow \infty$, for the same value of $\alpha_{N}$ as in Theorem 1.1.

The above correction means that SVP and SIVP are not equivalent on a positive proportion of cyclic lattices, however they are equivalent on a positive proportion of WR cyclic lattices spanned by rotations of a single vector. In other words, in the denominator of Eq. (18) in Remark $4.4, \mathcal{C}_{N}$ should be replaced by $\mathcal{R}_{N}$. Finally, in light of this correction, it makes sense to restate Question 2 in Sect. 6 of our paper as:

How many WR lattices are there among all $\tau$-invariant sublattices of $\mathbb{Z}^{N}$ for an arbitrary permutation $\tau \in S_{N}$ ?

\section{Reference}

1. Fukshansky, L., Sun, X.: On the geometry of cyclic lattices. Discrete Comput. Geom. 52(2), 240-259 (2014) 